\title{
Intrahepatic Cholestasis of Pregnancy - Report of two Cases
}

\author{
Dr. Kanupuru Manaswini ${ }^{1}$, Dr. Renuka ${ }^{2}$, Dr. K.Saraswathi ${ }^{3}$ \\ ${ }^{1}$ Junior Resident Dept $O f O B G$, \\ ${ }^{2}$ Assistant Professor Dept $O f O B G$, \\ ${ }^{3}$ Professor Dept Of OBG, Sreebalaji Medical College \&Hospital, CHENNAI.
}

\begin{abstract}
Intrahepatic cholestasis of pregnancy (ICP) also known as obstetric cholestasis, is a cholestatic disorder characterized by pruritus, elevated serum aminotransferases and bile acid levels with gradual onset in the second or third trimester of pregnancy may lead to adverse fetal outcome, and spontaneous relief of signs and symptoms within two to three weeks after delivery.

Keywords : Intrahepatic cholestasis of pregnancy, pruritis.
\end{abstract}

\section{Background}

ICP was originally described in 1883 by Ahlfeld as recurrent jaundice in pregnancy that resolved following delivery. Pruritus was not mentioned in this report, but in subsequent case reports published in the 1950s, severe pruritus with or without jaundice was reported in conjunction with the condition, in addition to complete resolution following delivery and high recurrence rates in subsequent pregnancies[1,2]

\section{Case 1:}

A 25 year old Primigravida with 38 weeks of gestation was admitted to antenatal ward with the complaints of severe itching all over the body, lack of sleep since one week . The clinical examination, revealed that client is weakly built, pale in appearance, not icteric, severe itching was evident which was not associated with secondary skin lesions. While her vital parameters remained normal. Ultrasonography showed a SLIUF corresponding to 38 weeks gestation and estimated fetal weight of 3245+/-220 gms. Urine report was negative for the presence of bile salts and pigments. Serum markers remained elevated with markable elevation of S. Bilirubin level 2.2mg/dL, SGOT - 46 IU/L, Alkaline phosphatase-287 IU/L, S. Albumin $2.98 \mathrm{~g} / \mathrm{dL}$, PT--- 14.0, aPTT--- 46.6, Hb---10.0 g/dL and peripheral smear report gave the impression of Normocytic normochromic anemia. Hepatitis B report showed negative . Medical Gastroentrologist opinion obtained regarding elevated liver enzymes she was treated with Ursodeoxycholic acid 300mg and other supportive drugs for itching advised serum bile acids, as serum bile acids was also elevated and her symptoms does'nt reduced. She was induced at 39 weeks, she delivered vaginally an alive male baby weighing $3.2 \mathrm{~kg}$ with apgar of 8/10,9/10.

\section{Case 2:}

A 33-year old second Gravida with 36 weeks of gestation was admitted to antenatal ward with complaints of itching of both hands and palms since 3 weeks. Clinical examination showed normal findings,vitals were stable. On admission ultrsonograhy showed a SLIUF corresponding to 36 weeks. Liver function test shows the elevated SGOT (180 IU/l) as well as SGPT (205 IU/l) levels in serum. The fasting total bile acids (TBA) level was $113.3 \mu \mathrm{mol} / 1$. Hepatitis B and C were negative. Treated with ursodeoxycholic acid, around 37 weeks she had reduced fetal movements and worsening of cardiotocographic test, the pregnancy was ended with elective caesarean section. An alive female baby delivered with birthweight of $2.6 \mathrm{~kg}$ with apgar of $8 / 10,9 / 10$.

\section{Discussion:}

The causes of intrahepatic cholestasis of pregnancy are still not fully understood. are likely to be important in the etiopathogenesis of the disease, multiple pregnancies which are associated with higher hormone levels, show a higher incidence of ICP, it resolves quickly after delivery,Treatment with progesterone in the third trimester of pregnancy has been shown to be associated with the development of ICP, While most pregnant women experience some itch from time to time, itching on the palms and soles without a visible rash, or persisting severe or extensive itch symptoms should be reported to obstetrican .To obtain a diagnosis of ICP, there are two LFT and Serum bile acid test. (LFTs) is a simple blood test, but only with elevated bile acid levels (however LFTs are not always elevated in ICP patients). The serum bile acid blood test for ICP is a quantitative measurement of bile salts. The results of this test often take longer to return, but the test is more specific for ICP.Other problems with the liver that occur in pregnancy like preeclamsia and HELLP syndrome should be considered. Furthermore, other causes of hepatitis, like hepatitis viruses, cancer and certain medications, should 
also be considered. Fetal demise due to ICP can occurs even in minute to hours even with a reactive nonstress test,[4,5] thus, fetal distress from ICP is not predicted by conventional methods of fetal surveillance. Animal studies have suggested two possible etiologies of sudden fetal demise associated with ICP. First, elevated bile acids may acutely impair the function of cardiomyocytes resulting in heart failure.[6] Alternatively, meconium staining may cause vasoconstriction of placental and umbilical blood vessels resulting in fetal acidosis. Rodrigues et al found that while maternal serum bile acid levels decrease with ursodiol treatment, the elevated concentration of bile acids in meconium remained unaltered.[5] This may explain why negative outcomes are associated with maximum bile acid levels during pregnancy, and improved laboratory values after treatment do not appear to alter the risk of acute fetal demise.[3] Furthermore, meconium staining complicates 80 to $100 \%$ of deliveries with ICP.Delivery by 35-37 completed weeks may be important to fetal outcome as a recent study demonstrated that in severe ICP (defined as bile acids greater than $40 \mathrm{umol} / \mathrm{L}$ ) the risk of stillbirth was $1.5 \%$ compared to $0.5 \%$ of uncomplicated pregnancies. This risk increases further if bile acids doubled[7].

\section{Conclusion}

The maternal cholestasis is transient with postnatal resolution, although affected women have increased rates of hepatobiliary disorders in later life. ICP is associated with adverse fetal outcomes. The risk of meconium-stained liquor, fetal asphyxia and spontaneous preterm delivery is greater in pregnancies with more marked elevations in maternal serum bile acid levels. The condition is also associated with IUD. The most effective pharmacological therapy for improvement of maternal symptoms and biochemical abnormalities is UDCA, and this has also been shown to reduce placental abnormalities and to improve placental bile acid transport in in vitro studies. Fetal outcomes are improved with a variety of strategies of active management, although the most effective intervention has not currently been established. A common practice is induction of labour at 37-38 wk of gestation with the aim of reducing the risk of IUD as many deaths occur at later gestations. Large therapeutic trials are required to establish which specific drug treatments or management strategies are effective at reducing the rates of adverse fetal outcomes.. The early detection of hepatic problem in pregnant women and implementation of proper treatment is crucial. In some cases of ICP the immediate termination of pregnancy by delivery induction or by cesarean section should be considered. The appropriate postpartum care of the mother and newborn is also important. The cooperation of medical experts team is required to improve the perinatal outcomes in women with ICP.

\section{References}

[1]. Svanborg A. A study of recurrent jaundice in pregnancy. ActaObstetGynecol Scand. 1954;33:434-444.[PubMed]

[2]. Thorling L. Jaundice in pregnancy; a clinical study. Acta Med Scand Suppl. 1955;302:1-123. [PubMed]

[3]. Glantz A, Marschall H-U, Mattsson L-A. Intrahepatic cholestasis of pregnancy: Relationships between bile acid levels and fetal complication rates. Hepatology 2004; 40 (2) 467-474

[4]. Lee RH, Incerpi MH, Miller DA, Pathak B, Goodwin TM. Sudden fetal death in intrahepatic cholestasis of pregnancy. ObstetGynecol 2009; 113 (2 Pt 2): 528-531

[5]. Rodrigues CMP, Marín JJG, Brites D. Bile acid patterns in meconium are influenced by cholestasis of pregnancy and not altered by ursodeoxycholic acid treatment. Gut 1999; 45 (3) 446-452

[6]. Williamson C, Gorelik J, Eaton BM, Lab M, de Swiet M, Korchev Y. The bile acid taurocholate impairs rat cardiomyocyte function: a proposed mechanism for intra-uterine fetal death in obstetric cholestasis. ClinSci (Lond) 2001; 100 (4) 363-369

[7]. Geenes V, Chappell LC, Seed PT, Steer PJ, Knight M, Williamson C. Association of Severe Intrahepatic Cholestasis of Pregnancy With Adverse Pregnancy Outcomes: A Prospective Population-Based Case-Control Study.Hepatology (Baltimore, Md). 2014;59(4):1482-1491. doi:10.1002/hep.26617. 